PUPT-1316

April 1992

\title{
Unitarity Relations in c=1 Liouville Theory
}

\author{
David A. Lowe \\ Joseph Henry Laboratories \\ Princeton University \\ Princeton, New Jersey 08544
}

\begin{abstract}
We consider the S-matrix of $c=1$ Liouville theory with vanishing cosmological constant. We examine some of the constraints imposed by unitarity. These completely determine $(N, 2)$ amplitudes at tree level in terms of the $(N, 1)$ amplitudes when the "plus" tachyon momenta take generic values. A surprising feature of the matrix model results is the lack of particle creation branch cuts in the higher genus amplitudes. In fact, we show the naive field theory limit of Liouville theory would predict such branch cuts. However, unitarity in the full string theory ensures that such cuts do not appear in genus one $(N, 1)$ amplitudes. We conclude with some comments about the genus one $(N, 2)$ amplitudes.
\end{abstract}




\section{INTRODUCTION}

The Liouville model of $c=1$ quantum gravity with vanishing cosmological constant describes a sum over Euclidean random surfaces coupled to a non-compact scalar boson. With this simple physical interpretation one would expect this to be a quantum theory described by a unitary S-matrix. Following Polyakov [1] and Minic and Yang [2] we consider the S-matrix defined by the resonant Liouville tachyon scattering amplitudes. Because the discrete states [1,3] appear as intermediate states at poles in these amplitudes it is necessary to include these in the Hilbert space of our theory, and hence in the S-matrix. This kind of S-matrix resembles what we see in more realistic string theories, i.e. amplitudes with poles due to onshell intermediate states. We may hope to obtain more direct insight into higher dimensional strings by studying this kind of theory. It should be noted that the S-matrices considered in matrix model work $[12,13,9]$ do not possess this property.

From the Liouville point of view the only closed string amplitudes that have been computed are the $(N, 1)$ tachyon amplitudes on the sphere [1-6], the genus one partition function $[7,8]$, and the three point couplings of discrete states on the sphere $[10,11]$. In this paper we use the unitarity constraints to extract information about some other correlation functions. We find that unitarity completely determines the $(N, 2)$ tachyon amplitudes on the sphere in terms of the $(N, 1)$ amplitudes when the "plus" tachyon momenta take generic values. We then consider the integral expression for genus one Liouville amplitudes. To leading order in the tachyon potential, the tachyon part of the field theory limit of these amplitudes is shown to correspond to a massless $\varphi^{3}$ theory with a time dependent coupling. This would lead one to expect log branch cuts in the momenta. We show unitarity conditions in the full string theory imply these cuts do not appear for the case of $(N, 1)$ amplitudes at genus one. We conclude with some comments about the genus one $(N, 2)$ amplitudes. 


\section{The S-Matrix And Unitarity}

Let us begin by reviewing the form of the tree-level tachyon amplitudes in resonant Liouville theory coupled to $c=1$ matter. Tachyon vertex operators take the form

$$
T(p)=\exp (i p X+(-1+\varepsilon) \phi)
$$

where $X$ is the matter field and $\phi$ the Liouville field. For positive (negative) chirality tachyons $\varepsilon=p(\varepsilon=-p)$. For $(N-1,1)$ amplitudes, i.e. $N-1$ positive chirality particles $T^{+}$and one negative chirality particle $T^{-}$the answer is $[1,4,5,16]$

$$
\left\langle T^{+}\left(p_{1}\right) \cdots T^{+}\left(p_{N-1}\right) T^{-}\left(p_{N}\right)\right\rangle=\frac{\pi^{N-3}}{(N-3) !} z\left(p_{1}\right) \cdots z\left(p_{N-1}\right)
$$

where $z(p)=\Gamma(1-2 p) / \Gamma(2 p)$. The derivation of this formula does not require us to restrict to $p_{i}>0$, so we take $p_{i} \in(-\infty, \infty)$ which corresponds to "wrongly" dressed tachyons for $p_{i}<0$. A similar formula holds for $(1, N-1)$ amplitudes with $z(p) \rightarrow z(-p)$. If we take the limit $p_{i} \rightarrow n / 2$ with $n$ a positive integer, the amplitude approaches a single pole coming from an on-shell intermediate state. Adopting an $i \epsilon$ prescription for the string propagator amounts to the replacement

$$
\frac{1}{L_{0}+\bar{L}_{0}-2} \rightarrow \frac{1}{L_{0}+\bar{L}_{0}-2+i \epsilon}
$$

So (1) will develop an imaginary part as a pole is approached corresponding to a shift $z(p) \rightarrow z(p+i \epsilon)$.

We now use the amplitudes of resonant Liouville theory to define a S-matrix to describe the scattering of tachyons and the discrete states. Here we are referring to the discrete states of the form $V_{j, m}^{ \pm}=P_{j, m}(X) P_{j, m}(\bar{X}) \exp (i m X+(-1 \pm j) \phi)$ in which $P_{j, m}(X)$ is a polynomial in the derivatives of $X$, and $j$ and $m$ are $S U(2)$ quantum numbers as discussed in [10]. 
In the $\mu=0$ theory we obtain a delta function expressing conservation of energy by Wick rotating the $\phi$ zero mode, $\phi_{0} \rightarrow i \phi_{0}$ and integrating along the real axis. This leads to a physical picture of particle scattering since now the tachyon wavefunctions look like $\exp \left(i \varepsilon \phi_{0}+i p X_{0}\right) . X_{0}$ is interpreted as the space direction and $\phi_{0}$ as time. + tachyons with $p>0$ then correspond to right moving in states while those with $p<0$ should be interpreted as left moving out states. Similarly - tachyons with $p<0$ are left moving in states, and $p>0$ - tachyons are right moving out states.

We choose the tachyon states to be normalized as

$$
\left\langle T\left(p^{\prime}\right) \mid T(p)\right\rangle=4 \pi|p| \delta\left(p-p^{\prime}\right),
$$

and the tachyon propagator is $i /\left(\varepsilon^{2}-p^{2}+i \epsilon\right)$. Decomposing the S-matrix as

$$
S=1+i R,
$$

we define the connected part of $R$ corresponding to tachyon scattering as

$$
\left\langle\prod_{i} T\left(p_{i}\right)\left|R_{c}\right| \prod_{j} T\left(p_{j}\right)\right\rangle=\pi\left\langle\prod_{i} T\left(p_{i}\right) \prod_{j} T\left(p_{j}\right)\right\rangle,
$$

where $\left\langle\prod T\left(p_{i}\right) \prod T\left(p_{j}\right)\right\rangle$ is the resonant Liouville scattering amplitude, and we have suppressed the delta function expressing conservation of energy and momentum. The factor of $\pi$ is fixed by demanding consistency of the unitarity relations with the normalizations implicit in (1) .

Unitarity is an important physical constraint for the consistency of our theory. Imposing $S S^{\dagger}=1$ gives

$$
R-R^{\dagger}=i R R^{\dagger}=i R^{\dagger} R,
$$


or equivalently

$$
2 \operatorname{Im}\left\langle\prod_{i} T\left(p_{i}\right)|R| \prod_{j} T\left(p_{j}\right)\right\rangle=\sum_{n}\left\langle\prod_{i} T\left(p_{i}\right)|R| n\right\rangle\left\langle n|R| \prod_{j} T\left(p_{j}\right)\right\rangle,
$$

where we sum over all positive energy intermediate states allowed by overall conservation of energy and momentum. For tachyon intermediate states this boils down to replacing the tachyon propagator by $2 \pi \delta\left(\varepsilon^{2}-p^{2}\right) \theta(\varepsilon)$. For discrete states the propagator is replaced by $2 \pi \delta\left(\varepsilon^{2}-p^{2}-M^{2}\right) \theta(\varepsilon) \mathcal{P}_{M}$ for a discrete state of mass $M . \mathcal{P}_{M}$ is an operator which projects onto the physical discrete values of $\varepsilon$ and $p$ for a state of mass $M$.

\section{3. $(\mathrm{N}, 2)$ Amplitudes on the Sphere}

We now prove that the relation (7) is sufficient to determine all tree-level $(N, 2)$ amplitudes when the + tachyons take generic values of momenta. Energy and momentum conservation give

$$
\begin{aligned}
& \sum p_{i}=N / 2, \\
& \sum k_{i}=-N / 2 .
\end{aligned}
$$

Here $p_{i}$ refer to + tachyons and $k_{i}$ to - tachyons. It has been argued in [16] that these amplitudes should vanish up to delta function terms. By considering the integral representation it may be seen that these delta function terms arise due to on-shell intermediate + tachyons as the $k_{i} \rightarrow-n_{i} / 2$. Here $n_{i}$ is a positive integer. There is no contribution from the discrete states as long as the $p_{i}$ are kept at generic values.

For a general $(N, 2)$ amplitude a typical diagram is shown in Fig 1. Summing over the permutations of $k_{1}$ and $k_{2}$ eliminates the $\theta(\varepsilon)$ factor from the on-shell tachyon propagator. This leaves us with a sum over the subdivisions $S_{1}, S_{2}$ of the set $S$ of + tachyon momenta which satisfy $\left|S_{1}\right|=n$. Let $q(S)$ denote the sum over 
the momenta in set $S$. The unitarity relations (7) in the limit $k_{1} \rightarrow-n / 2$ then lead to

$$
\begin{aligned}
& 2 \pi \operatorname{Im}\left\langle T^{+}\left(p_{1}\right) \cdots T^{+}\left(p_{N}\right) T^{-}\left(k_{1}\right) T^{-}\left(k_{2}\right)\right\rangle \\
& =\sum_{\substack{S_{1} \sqcup S_{2}=S \\
\left|S_{1}\right|=n}} 2 \pi^{3}\left\langle\prod_{S_{1}} T^{+}\left(p_{i}\right) T^{+}\left(-q\left(S_{1}\right)-k_{1}\right) T^{-}\left(k_{1}\right)\right\rangle \delta\left(\left(n+2 k_{1}\right)\left(n-2 q\left(S_{1}\right)\right)\right) \\
& \quad \times\left\langle T^{+}\left(q\left(S_{1}\right)+k_{1}\right) \prod_{S_{2}} T^{+}\left(p_{j}\right) T^{-}\left(k_{2}\right)\right\rangle \\
& =\sum_{\substack{S_{1} \sqcup S_{2}=S \\
\left|S_{1}\right|=n}} \frac{2 \pi^{N+1}}{(n-1) !(N-n-1) !} z\left(p_{1}\right) \cdots z\left(p_{N}\right) z\left(q\left(S_{1}\right)+k_{1}\right) z\left(-q\left(S_{1}\right)-k_{1}\right) \\
& \quad \times \delta\left(\left(2 q\left(S_{1}\right)-n\right)\left(n+2 k_{1}\right)\right) \\
& =\sum_{\substack{S_{1} \sqcup S_{2}=S \\
\left|S_{1}\right|=n}}-\frac{4 \pi^{N+1}}{(n-1) !(N-n-1) !} z\left(p_{1}\right) \cdots z\left(p_{N}\right)\left|q\left(S_{1}\right)+k_{1}\right| \delta\left(n+2 k_{1}\right),
\end{aligned}
$$

where we used the fact that $z(q) z(-q)=-4 q^{2}$. In fact we can see that the real part of this amplitude should vanish. Factorizing the amplitude in the limit $k_{1} \rightarrow-n / 2$ and keeping only the real part we obtain

$$
\begin{aligned}
& \sum_{\substack{S_{1} \sqcup S_{2}=S \\
\left|S_{1}\right|=n}}\left\langle\prod_{S_{1}} T^{+}\left(p_{i}\right) T^{+}\left(-q\left(S_{1}\right)-k_{1}\right) T^{-}\left(k_{1}\right)\right\rangle \frac{1}{\left(n+2 k_{1}\right)\left(n-2 q\left(S_{1}\right)\right)} \\
& \times\left\langle T^{+}\left(q\left(S_{1}\right)+k_{1}\right) \prod_{S_{2}} T^{+}\left(p_{j}\right) T^{-}\left(k_{2}\right)\right\rangle \\
= & \sum_{\substack{S_{1} \sqcup S_{2}=S \\
\left|S_{1}\right|=n}} z\left(p_{1}\right) \cdots z\left(p_{N}\right) \frac{\pi^{N-2}}{(n-1) !(N-n-1) !} \frac{z\left(q\left(S_{1}\right)+k_{1}\right) z\left(-q\left(S_{1}\right)-k_{1}\right)}{\left(n+2 k_{1}\right)\left(n-2 q\left(S_{1}\right)\right)} \\
= & z\left(p_{1}\right) \cdots z\left(p_{N}\right) \frac{2 \pi^{N-2}}{(n-1) !(N-n-1) !} \frac{N !}{n !(N-n) !} \frac{k_{1}+\frac{n}{N} \sum p_{i}}{n+2 k_{1}} .
\end{aligned}
$$

Recalling $\sum p_{i}=N / 2$ we see the real part of the residue of the pole vanishes as $k_{1} \rightarrow-n / 2$, so the imaginary part (9) is the full amplitude. 
Let us compare this to the matrix model predictions for non-resonant amplitudes at tree-level $[4,5,12,13,17]$

$$
\begin{aligned}
& \left\langle T^{+}\left(p_{1}\right) \cdots T^{+}\left(p_{N}\right) T^{-}\left(k_{1}\right) \cdots T^{-}\left(k_{M}\right)\right\rangle= \\
& \quad \mu^{s}(-\pi)^{N+M-3} z\left(p_{1}\right) \cdots z\left(p_{N}\right) z\left(-k_{1}\right) \cdots z\left(-k_{M}\right) P\left(\left\{p_{i}\right\},\left\{k_{j}\right\}\right),
\end{aligned}
$$

where $P\left(\left\{p_{i}\right\},\left\{k_{j}\right\}\right)$ is a polynomial in the momenta and $s=\sum p_{i}-\sum k_{j}+2-$ $N-M$. First we recall how the $\phi$ zero mode integrations are related. In the case of non-resonant amplitudes we have [6]

$$
\int_{-\infty}^{\infty} d \phi_{0} \exp \left(s \phi_{0}-\Delta e^{-\phi_{0}}\right)=\Delta^{s} \Gamma(-s)
$$

if we take $\Delta>0$. Here $\Delta$ is the bare cosmological constant, related to the renormalized cosmological constant appearing in (11) by $\Delta=\mu \log (\mu)$ as discussed in [17]. Alternatively we may take $\phi \exp (-\phi)$ to be the cosmological constant operator. We see therefore that the energy conserving delta function $\delta(s)$ of the resonant amplitude is replaced by a pole as $s \rightarrow 0$. The rule for comparing the resonant amplitudes and the $s \rightarrow 0$ limit of the non-resonant amplitudes is then: extract the residue of the $-1 / s$ pole and insert the appropriate $i \epsilon$ terms coming from the shifted string propagator.

For the $(N, 1)$ case this is straightforward. Kinematics fixes $k_{1}=-(N-1+s) / 2$ so the $-1 / s$ pole comes from the $z\left(-k_{1}\right)$ factor in $(11)$. For the general $(N, M)$ amplitude with $N>1$ and $M>1$ there is no $-1 / s$ pole unless we impose an extra constraint on the momenta which will give rise to nonanalytic terms. Therefore these amplitudes are usually analytically continued to zero.

Let us analyze the nonanalytic contributions for $(N, 2)$ amplitudes when the $p_{i}$ take generic values. In the limit $s \rightarrow 0$ and $k_{1} \rightarrow-n / 2$ (with $n$ a positive integer) (11) will lie on a double pole

$$
\begin{aligned}
& \left\langle T^{+}\left(p_{1}\right) \cdots T^{+}\left(p_{N}\right) T^{-}\left(k_{1}\right) T^{-}\left(k_{2}\right)\right\rangle=\mu^{s} \pi^{N-1} z\left(p_{1}\right) \cdots z\left(p_{N}\right) P\left(\left\{p_{i}\right\},\left\{k_{j}\right\}\right) \\
& \times \frac{1}{((n-1) !(N-n-1) !)^{2}}\left(\frac{1}{2 k_{1}+n}+\frac{1}{-2 k_{1}-n-s}\right) \frac{1}{-s} .
\end{aligned}
$$


Inserting the appropriate $i \epsilon$ terms in the factors multiplying $-1 / s$ we obtain for the resonant amplitude

$$
-\pi^{N-1} z\left(p_{1}\right) \cdots z\left(p_{N}\right) P\left(\left\{p_{i}\right\},\left\{k_{j}\right\}\right) \frac{1}{((n-1) !(N-n-1) !)^{2}} 2 \pi i \delta\left(n+2 k_{1}\right) .
$$

Therefore we see that the real part of the amplitude vanishes and the imaginary part should agree with that computed using the unitarity relations (9) . This has been checked for the four and five point functions. For example as $k_{1} \rightarrow-1 / 2$ the resonant $(3,2)$ amplitude in any kinematic configuration can be written

$$
\begin{aligned}
& \left\langle T^{+}\left(p_{1}\right) T^{+}\left(p_{2}\right) T^{+}\left(p_{3}\right) T^{-}\left(k_{1}\right) T^{-}\left(k_{2}\right)\right\rangle= \\
& -2 \pi^{3} i z\left(p_{1}\right) z\left(p_{2}\right) z\left(p_{3}\right)\left(\left|p_{1}+k_{1}\right|+\left|p_{2}+k_{1}\right|+\left|p_{3}+k_{1}\right|\right) \delta\left(1+2 k_{1}\right),
\end{aligned}
$$

in agreement with (9) .

\section{Tachyon Effective Field Theory}

Now we investigate the tachyon effective action that follows from the field theory limit of the one loop correlators. The leading term in the tachyon potential will turn out to be a simple cubic. This would lead us to expect log branch cuts in the momenta at one loop in obvious disagreement with matrix model results. We will show however that the unitarity relations imply these cuts are absent in the full string theory indicating a very subtle interplay between the tachyon field and the discrete states in this kind of effective theory.

Consider then the formula for the genus one amplitudes in Liouville theory of tachyon operators of the form $\exp (i p X+(-1+\varepsilon) \phi)$

$$
\int_{\mathcal{F}} \frac{d^{2} \tau}{\tau_{2}^{2}} \prod_{i=1}^{N} \int d^{2} z_{i} \prod_{1 \leq j<i} \chi\left(z_{i}-z_{j} \mid \tau\right)^{-4 \mathbf{k}_{i} \cdot \mathbf{k}_{j}}
$$

with

$$
\chi(z \mid \tau)=\exp \left(\frac{-\pi(\operatorname{Im} z)^{2}}{\tau_{2}}\right)\left|\frac{\theta_{1}(z \mid \tau)}{\theta_{1}^{\prime}(0 \mid \tau)}\right|
$$


and

$$
\mathbf{k}=(-1+\varepsilon, k), \quad \mathbf{k}_{i} \cdot \mathbf{k}_{j}=\left(-1+\varepsilon_{i}\right)\left(-1+\varepsilon_{j}\right)-k_{i} k_{j}
$$

where $\tau_{2}=\operatorname{Im} \tau$ and $\mathcal{F}$ is the fundamental region of the modular group $\tau_{2}>0$, $|\tau|>1$ and $-1 / 2 \leq \operatorname{Re} \tau<1 / 2$. We may take the field theory limit by sending the lower cutoff to zero and extracting the leading asymptote of the integrand as $\tau_{2} \rightarrow \infty$. This should correspond to keeping only the purely tachyonic contribution to $(16)$. For $z=x+\tau y$, and $y$ fixed as $\tau_{2} \rightarrow \infty$,

$$
\chi(z \mid \tau) \rightarrow \exp \left(\pi \tau_{2} y(1-y)\right)|1-\exp (2 \pi i z)|
$$

When taking the field theory limit we discard the $\exp (2 \pi i z)$ term which is suppressed with respect to the leading term except when $y \rightarrow 0$, when "contact" terms appear. This corresponds to only keeping the ring diagram contribution. Substituting (19) into the genus one integral and trivially doing the $\tau_{1}$ and $x_{i}$ integrals we get

$$
\int_{0}^{\infty} d \tau_{2} \tau_{2}^{N-2} \prod_{i=1}^{N} \int_{0}^{1} d y_{i} \exp \left(-4 \pi \tau_{2} \sum_{i<j}\left|y_{i}-y_{j}\right|\left(1-\left|y_{i}-y_{j}\right|\right) \mathbf{k}_{i} \cdot \mathbf{k}_{j}\right)
$$

For this to be well defined we must pick a specific ordering of the $y_{i}$, lets say $y_{1}<y_{2}<\cdots<y_{N}$, and continue the $k_{i}$ to a region where the integral converges.

Now we change variables to the Feynman parameters

$$
\begin{aligned}
\alpha_{i} & =y_{i+1}-y_{i}, \quad i<N \\
\alpha_{N} & =1-y_{N}+y_{1} .
\end{aligned}
$$

The term in the exponential becomes

$$
\sum_{i<j}\left(y_{j}-y_{i}\right)\left(1-\left(y_{j}-y_{i}\right)\right) \mathbf{k}_{i} \cdot \mathbf{k}_{j}=\sum_{i<j} \alpha_{i} \alpha_{j} \beta_{i j}
$$


where

$$
\beta_{i j}=\sum_{\substack{l>i, m>j \\ m>l, l \leq j}} \mathbf{k}_{l} \cdot \mathbf{k}_{m}+\sum_{\substack{l \leq i, m \leq j \\ m>l, m>i}} \mathbf{k}_{l} \cdot \mathbf{k}_{m}
$$

and we finally get

$$
\int_{0}^{\infty} d \tau_{2} \tau_{2}^{N-2} \prod_{i=1}^{N} \int_{0}^{1} d \alpha_{i} \delta\left(\sum \alpha_{i}-1\right) \exp \left(-4 \pi \tau_{2} \sum_{l<m} \alpha_{l} \alpha_{m} \beta_{l m}\right)
$$

which is just the proper time representation for the ring graph of a massless $\varphi^{3}$ theory with a time dependent coupling $g_{s t}=g_{0} e^{-\phi}$. Thus to leading order in the tachyon potential the effective action for the tachyon field for zero cosmological constant is

$$
S=\frac{1}{2 g_{0}^{2}} \int d x d \phi e^{2 \phi}\left(\left(\partial_{x} T\right)^{2}+\left(\partial_{\phi} T\right)^{2}-T^{2}-\frac{1}{3} T^{3}\right)
$$

which is familiar form from the work of $[15,16]$. Naturally there will be higher order corrections to the potential $V(T)$ and interaction terms with the discrete states but if we only consider the cubic interaction term we expect to see logarithmic cuts in the momenta at higher genus. For example evaluating the three point ring diagram we find a branch cut that goes like $\log (1-2 p) \theta(1-2 p) /|1-2 p|$ as $p \rightarrow \frac{1}{2}$. These cuts are not observed in the matrix model and we would like to understand their absence from the Liouville point of view.

\section{Absence of Branch Cuts at Genus One}

Let us apply the unitarity relations $(7)$ to the $(N, 1)$ amplitudes at genus one. Fig 2 shows the type of diagram to be considered. We take the momenta of the + external tachyons $p_{i}$ to be generic. Branch cuts will then appear as finite contributions to the discontinuity in the imaginary part of the amplitude. Note that when some of the $p_{i}$ lie at discrete values delta function terms will appear corresponding to poles in the full amplitude, however this is the same situation as at tree-level. 
Let us consider the different possible combinations of intermediate states. Two + tachyons are forbidden by kinematics. We have already seen from the form of the tree level amplitudes that when - tachyons appear as intermediate states they will be forced to lie at discrete values of momenta. In fact, the situation when both the intermediate states are discrete is also forbidden by kinematics as long as the $p_{i}$ are generic.

The only case to consider then is when one + tachyon and one discrete state appears. The configuration consistent with kinematics is when the vertices are

$$
\begin{aligned}
& \left\langle V_{j,-m}^{-} T^{+}(-q) T^{+}\left(p_{i_{1}}\right) \cdots T^{+}\left(p_{i_{n}}\right) T^{-}\left(k_{1}\right)\right\rangle \quad \text { and } \\
& \left\langle V_{j, m}^{+} T^{+}(q) T^{+}\left(p_{i_{n+1}}\right) \cdots T^{+}\left(p_{i_{N}}\right)\right\rangle .
\end{aligned}
$$

These discrete state correlation functions may be obtained simply by factorizing the known results for $(N, 1)$ tachyon correlators. This tells us the first vertex vanishes while the second is finite. No additional divergence will arise from the $q$ integration implicit in (7) so this contribution to the imaginary part vanishes.

This proves that particle creation branch cuts are absent in $(N, 1)$ amplitudes at genus one. It is natural to expect this to be a general feature of $(N, 1)$ amplitudes at arbitrary genus. Unexpectedly the branch cuts observed in the naive effective tachyon field theory are absent in the full string theory. This result places strong constraints on possible string field theories derived from the Liouville point of view, and provides further evidence for the equivalence of the $c=1$ matrix model and Liouville theory coupled to $c=1$ conformal matter.

Finally, consider the $(N, 2)$ amplitudes at genus one. In this case the vertices appearing in the unitarity relations may include some rather singular discrete state correlation functions which are not well understood. However, only considering the intermediate state with two + tachyons one might expect a finite imaginary part leading to a branch cut. Let us analyze this case, shown in Fig 3. Define $n$ to be the number of incoming + tachyons on the left. Kinematics fixes $k_{1}=-(n+1) / 2$ 
on-shell, and we also have

$$
q^{\prime}=\sum_{i=1}^{n} p_{i}+k_{1}-q, \quad \quad \varepsilon^{\prime}=\sum_{i=1}^{n} p_{i}-\varepsilon-k_{1}-n-1 .
$$

The discontinuity in the imaginary part of the amplitude coming from this diagram is

$$
\begin{aligned}
\text { Disc } & =\frac{\pi^{N+1}}{n !(N-n) !} z\left(p_{1}\right) \cdots z\left(p_{N}\right) \\
& \times \int d q^{2} \delta\left(\varepsilon^{2}-q^{2}\right) \theta(\varepsilon) \delta\left(\varepsilon^{\prime 2}-q^{\prime 2}\right) \theta\left(\varepsilon^{\prime}\right) z(q) z(-q) z\left(q^{\prime}\right) z\left(-q^{\prime}\right) \\
& =\frac{\pi^{N+1}}{n !(N-n) !} z\left(p_{1}\right) \cdots z\left(p_{N}\right) \int d q 8 q q^{\prime 2} \\
& \times \theta(q) \theta\left(\sum_{i=1}^{n} p_{i}-q-k_{1}-n-1\right) \delta\left(\left(2 \sum_{i=1}^{n} p_{i}-2 q-n-1\right)\left(2 k_{1}+n+1\right)\right) .
\end{aligned}
$$

A finite imaginary part might come from integrating out the delta function. This sets $q^{\prime}=k_{1}+(n+1) / 2$ which vanishes on-shell causing this contribution to the discontinuity to also vanish. The presence of the $z$ factors, in particular the decoupling of the zero momentum mode $(z(0)=0)$, is crucial here.

Note that there is a contribution to the discontinuity proportional to $\delta\left(2 k_{1}+\right.$ $n+1)$

$$
\text { Disc }=\frac{2 \pi^{N+1}}{3 n !(N-n) !} z\left(p_{1}\right) \cdots z\left(p_{N}\right)\left(\sum_{i=1}^{n} p_{i}+k_{1}\right)^{3} \delta\left(2 k_{1}+n+1\right),
$$

which is of the form that would be expected from the resonant limit of the matrix model prediction for this amplitude, namely a polynomial in the momenta multiplied by the product of $z$ factors (the $\delta\left(2 k_{1}+n+1\right)$ factor arises in the same way as in the $(N, 2)$ amplitudes on the sphere as described above). It will, of course, be necessary to include the full sum over intermediate states to see complete agreement between the polynomial terms. 


\section{Conclusion}

We have examined some of the constraints imposed by unitarity on the S-matrix of $c=1$ Liouville theory. These determined the $(N, 2)$ amplitudes on the sphere in a certain resonant limit and allowed us to explain the absence of branch cuts in some genus one amplitudes from the Liouville point of view. Unitarity should also allow us to derive interesting relations between higher genus amplitudes, and may point the way to an off-shell sewing theorem, but this must await a better understanding of the role of the discrete states.

Acknowledgements: I thank A.M. Polyakov and I.R. Klebanov for many useful discussions and suggestions. This research was supported in part by DOE grant DE-AC02-76WRO3072, NSF grant PHY-9157482, and James S. McDonnell Foundation grant No. 91-48.

\section{REFERENCES}

1. A. M. Polyakov, Mod. Phys. Lett. A6 (1991) 635.

2. D. Minic and Z. Yang, Texas preprint UTTG-23-91.

3. D.J. Gross, I.R. Klebanov and M.J. Newman, Nucl. Phys. B350 (1991) 621.

4. P. Di Francesco and D. Kutasov, Phys. Lett. 261B (1991) 385.

5. P. Di Francesco and D. Kutasov, Princeton preprint PUPT-1276.

6. M. Goulian and M. Li, Phys. Rev. Lett. 66 (1991) 2051.

7. A. Gupta, S. P. Trivedi, and M. B. Wise, Nucl. Phys. B340 (1990) 475.

8. M. Bershadsky and I. R. Klebanov, Phys. Rev. Lett. 65 (1990) 3088; N. Sakai and Y. Tanii, Int. Jour. Mod. Phys. A6 (1991) 2743.

9. D.J. Gross and I.R. Klebanov, Nucl. Phys. B359 (1991) 3.

10. I.R. Klebanov and A.M. Polyakov, Mod. Phys. Lett. A6 (1991) 3273. 
11. Y. Matsumura, N. Sakai and Y. Tanii, Tokyo preprint TIT/HEP-186, STUPP-92-124.

12. G. Moore, Rutgers preprint RU-91-12 (1991); G. Moore, R. Plesser, and S. Ramgoolam, Yale preprint YCTP-P35-91; G. Moore and R. Plesser, Yale preprint YCTP-P7-92.

13. J. Polchinski, Nucl. Phys. B346 (1990) 253; S. R. Das and A. Jevicki, Mod. Phys. Lett. A5 (1990) 1639.

14. D. J. Gross and I. R. Klebanov, Nucl. Phys. B344 (1990) 475.

15. J. Polchinski, Nucl. Phys. B346 (1990) 253.

16. A. M. Polyakov, Princeton preprint PUPT-1289

17. I.R. Klebanov, Princeton preprint PUPT-1271. 